\title{
Control Of Current And Voltage Oscillations In A Short Dc Discharge Making Use Of External Auxiliary Electrode
}

\author{
A. S. Mustafaev
}

V.I. Demidov

I. Kaganovich

S. F. Adams

M. E. Koepke

See next page for additional authors

Follow this and additional works at: https://researchrepository.wvu.edu/faculty_publications

\section{Digital Commons Citation}

Mustafaev, A. S.; Demidov, V. I.; Kaganovich, I.; Adams, S. F.; Koepke, M. E.; and Grabovskiy, A., "Control Of Current And Voltage Oscillations In A Short Dc Discharge Making Use Of External Auxiliary Electrode" (2012). Faculty Scholarship. 493.

https://researchrepository.wvu.edu/faculty_publications/493 
Authors

A. S. Mustafaev, V. I. Demidov, I. Kaganovich, S. F. Adams, M. E. Koepke, and A. Grabovskiy 


\section{Control of current and voltage oscillations in a short dc discharge making use of external auxiliary electrode}

A. S. Mustafaev, V. I. Demidov, I. Kaganovich, S. F. Adams, M. E. Koepke, and A. Grabovskiy

Citation: Review of Scientific Instruments 83, 103502 (2012); doi: 10.1063/1.4757111

View online: https://doi.org/10.1063/1.4757111

View Table of Contents: http://aip.scitation.org/toc/rsi/83/10

Published by the American Institute of Physics

\section{Articles you may be interested in}

Modeling a short dc discharge with thermionic cathode and auxiliary anode

Physics of Plasmas 20, 101605 (2013); 10.1063/1.4823464

Electric probes for plasmas: The link between theory and instrument

Review of Scientific Instruments 73, 3409 (2002); 10.1063/1.1505099

Sharp transition between two regimes of operation of dc discharge with two anodes and thermionic emission from cathode

Physics of Plasmas 21, 053508 (2014); 10.1063/1.4876928

Suprathermal electron energy spectrum and nonlocally affected plasma-wall interaction in helium/air microplasma at atmospheric pressure

Physics of Plasmas 23, 103508 (2016); 10.1063/1.4964721

Nonlocal effects in a bounded low-temperature plasma with fast electrons

Physics of Plasmas 14, 057101 (2007); 10.1063/1.2436470

Understanding Langmuir probe current-voltage characteristics

American Journal of Physics 75, 1078 (2007); 10.1119/1.2772282
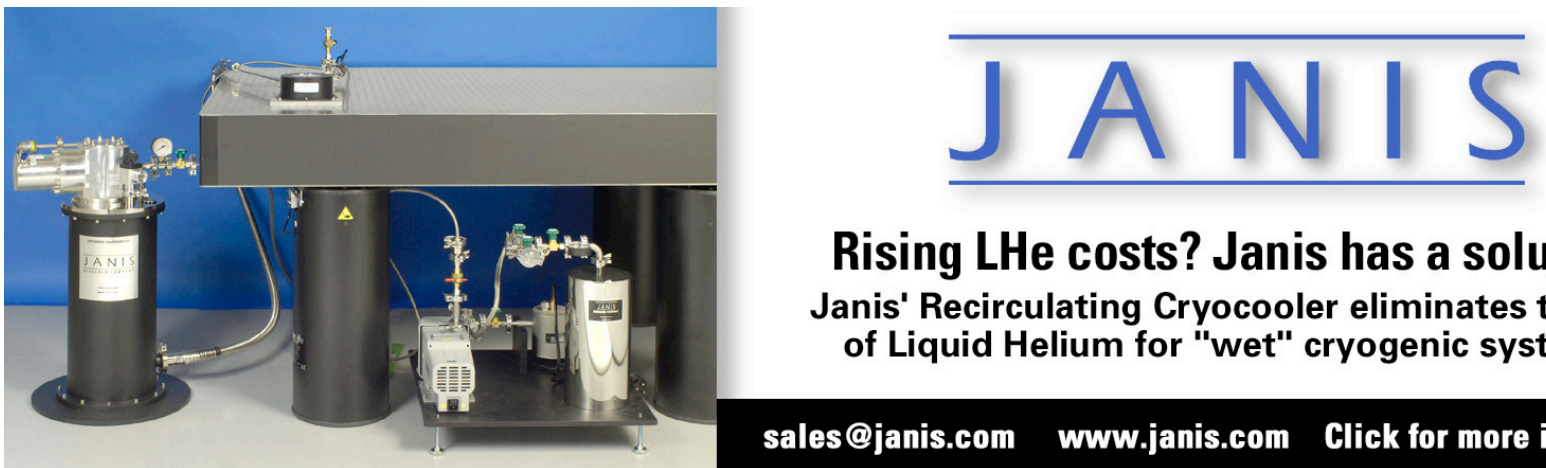

Rising LHe costs? Janis has a solution. Janis' Recirculating Cryocooler eliminates the use of Liquid Helium for "wet" cryogenic systems. 


\title{
Control of current and voltage oscillations in a short dc discharge making use of external auxiliary electrode
}

\author{
A. S. Mustafaev, ${ }^{1}$ V. I. Demidov, ${ }^{2}$ I. Kaganovich, ${ }^{3}$ S. F. Adams, ${ }^{4}$ M. E. Koepke, ${ }^{2}$ \\ and A. Grabovskiy ${ }^{1}$ \\ ${ }^{1}$ National Mineral Resources University (University of Mines), Saint-Petersburg 199106, Russia \\ ${ }^{2}$ West Virginia University, Morgantown, West Virginia 26506, USA \\ ${ }^{3}$ Princeton Plasma Physics Laboratory, Princeton, New Jersey 08543, USA \\ ${ }^{4}$ Air Force Research Laboratory, WPAFB, Ohio 45433, USA
}

(Received 30 July 2012; accepted 17 September 2012; published online 8 October 2012)

\begin{abstract}
A dc discharge with a hot cathode is subject to current and voltage plasma oscillations, which have deleterious effects on its operation. The oscillations can be inhibited by installing an auxiliary electrode, placed outside of anode. By collecting a modest current through a small opening in anode, we show that the discharge becomes stable, in a certain pressure range. This method of avoiding current oscillations can be used, for example, for high current stabilizers. (c) 2012 American Institute of Physics. [http://dx.doi.org/10.1063/1.4757111]
\end{abstract}

Gas-discharge plasma devices are widely used in plasma applications. ${ }^{1}$ A better understanding of the physics behind these devices has been developed over the last few decades and has allowed for the creation of discharge plasmas with more controllable parameters, including modification of the charged particle densities, temperatures (average energies), and electron energy distribution functions (EEDF). However, improving the performance of these new plasma tools is still a significant challenge for plasma engineering. Exploitation of nonlocal plasma properties allows additional dimensions and flexibility in adjusting plasma parameters. A remarkable property of such plasmas is that changing conditions in one place may lead to unexpected changes far away in another part of the plasma. Additionally, plasmas with nonlocal EEDF ${ }^{2,3}$ allow independent and effective managing of electrons belonging to different energy ranges. ${ }^{4,5}$ This, in turn, allows modification of the plasma properties in desirable ways, because different energetic groups of electrons are responsible for different processes, and their density modifications yield control over corresponding plasma processes.

One example of such a device with nonlocal plasma properties is a short dc discharge (several millimeters in length at the pressure of a few Torr, 10-100 $\mu \mathrm{m}$ for atmospheric pressure). ${ }^{6}$ The discharge consists of the cathode and anode sheathes and a negative glow plasma without a positive column. The plasma is created by the energetic electrons emitted by the cathode and accelerated by the near-cathode sheath to the energies above the ionization potential for the gas atoms. Inelastic collisions of the energetic electrons with atoms create slow thermal electrons and ions. A typical dimension of the discharge, $L$, is less than electron energy relaxation length, $\lambda_{\varepsilon}$, which for the noble gases and electrons with energies in elastic collision range (electron energy is below the first excitation potential), is typically on the order of $10 / p \mathrm{~cm}$, where $p$ is the gas pressure in Torr. ${ }^{2,3}$ For atmospheric pressures, it is found that $\lambda_{\varepsilon} \approx 100 \mu \mathrm{m}$ and $L<\lambda_{\varepsilon}$ for typical microdischarges. The short dc discharges, including microdischarges, can be used, for instance, for plasma-chemical and surface modification applications, ${ }^{7}$ as well as, for light sources, ${ }^{8}$ analytical sensors, ${ }^{9,10}$ and plasma electronic devices. ${ }^{11}$ In contrast to semiconductor devices, plasma discharges can be used under harsh conditions related, for example, to high temperatures and radiation levels of damaged nuclear plants (such as Fukushima-like disasters).

It is known (see, for example, Refs. 12-17) that dc electric discharges can be unstable with respect to excitation of various types of oscillations and instabilities. Oscillations are excited due to ionization plasma instability related to the falling volt-ampere characteristic of the discharge or part of it (see Refs. 12 and 18). These oscillations are affected by external electric circuits. There are many regimes of oscillations, which can be identified. While plasma instabilities, in principle, can be harnessed for some purposes, for example, for the generation of voltage oscillations, they are harmful for many other applications, for instance, for the development of current and voltage stabilization devices, and, in this case, they should be suppressed. Mitigation of these oscillations may not be a very simple task to accomplish. In this paper, we demonstrate how it is possible to achieve reliable suppression of the discharge oscillations by making use of an external auxiliary electrode. The method works for short dc discharge with thermal emission cathode in the nonlocal pressure regime $L<\lambda_{\varepsilon}$ $\approx 10 / p \mathrm{~cm}$.

An additional electron loss caused by the external electrode changes the voltage-ampere characteristic from falling to increasing with current. That makes the discharge stable and suppresses onset of the oscillations. It was experimentally demonstrated that a placement of the auxiliary electrode outside the discharge and subtraction of the anode current through a small opening in the anode provided the desirable effect.

The experimental device for manifestation of the above effect is shown schematically in Fig. 1. The plasma was created by the discharge, which existed between a grounded heated cathode and a positively biased anode. The cathode was a disc with a diameter of $1.0 \mathrm{~cm}$. The molybdenum 


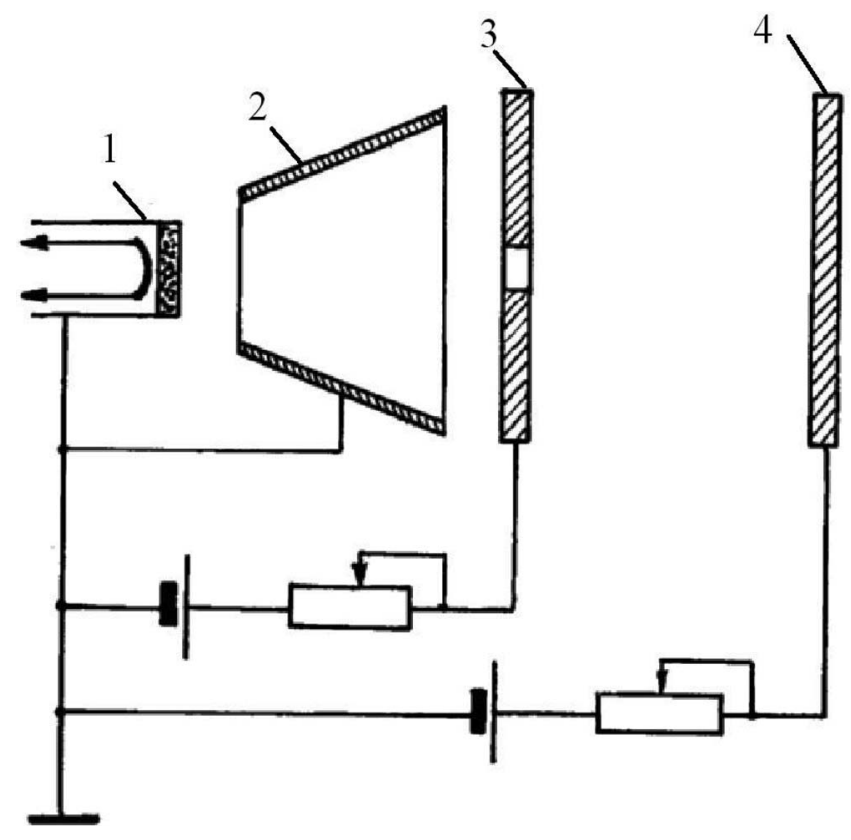

FIG. 1. Schematic diaphragm of the experimental device. Discharge takes place between cathode (1) and anode with a small opening (3) and is restricted radially by a cone screen (2). Auxiliary electrode (4) can provide the discharge plasma stabilization.

anode with an external diameter of $3.0 \mathrm{~cm}$ had a thickness of $0.2 \mathrm{~cm}$ and a central opening with an internal diameter of $0.2 \mathrm{~cm}$. The molybdenum auxiliary electrode had a diameter of $3.0 \mathrm{~cm}$ and was placed near the outer part of the anode. The distance between the cathode and anode was $0.8 \mathrm{~cm}$, and the distance between the anode and the auxiliary electrode could be changed from 0.1 to $5 \mathrm{~cm}$. A conical electrode (screen) restricted the discharge plasma in the radial direction. The conical screen was electrically connected to the cathode and, therefore, it was also grounded. Electrons emitted from the cathode were accelerated by the cathode fall and moved towards the anode. Because the cross sectional area of the opening was small compared to the anode cross sectional area, the energetic electrons were lost mostly on the anode and not in the opening. A cylindrical movable tantalum electric probe (not shown in Fig. 1) has been used to measure the EEDF, plasma potential, and plasma density. ${ }^{19,20}$ The probe had a diameter of $0.07 \mathrm{~mm}$ and length of $1 \mathrm{~mm}$. It was introduced into the plasma, perpendicular to the axis of the device, for making measurements in the interelectrode gap. It could also be used for the measurements near the opening. The measurements were conducted in spectrally pure helium at pressures from 1 to 10 Torr with a discharge current up to $5 \mathrm{~A}$.

In Fig. 2, the typical current-voltage experimental characteristics of the cathode-anode gap are presented. Curves 1, 2 , and 3 correspond to the case without collection of the current by the auxiliary electrode (diode regime). It is possible to see from Fig. 2 that in the diode regime, the discharge has a positive discharge differential resistance for the pressures below 1 Torr (curve 1). Increasing the gas pressure to 1 Torr leads to transformation of the discharge differential resistance to the slightly negative one (curve 2). Further increase of the

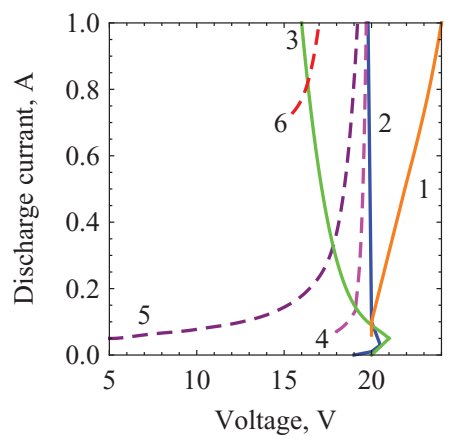

FIG. 2. The discharge current-voltage characteristics for helium gas pressure of 0.6 Torr (1), 1 Torr (2), and 4 Torr (3). The diode regime is shown by solid curves (no current to the auxiliary electrode). Regimes with the auxiliary electrode are shown by the dashed curves for helium gas pressure of 1 Torr and different currents to the auxiliary electrode (0.1 A (4) and $0.4 \mathrm{~A}$ (5)). Curve (6) is for helium gas pressure of 4 Torr and current to the auxiliary electrode of $0.1 \mathrm{~A}$.

gas pressure yields higher negative discharge differential resistance of the discharge (curve 3). The presence of the negative discharge differential resistance may lead to plasma instabilities and oscillations of the discharge voltage and current. For gas pressures between 1 and 2 Torr when the discharge differential resistance was negative, voltage oscillations have wide-band spectra and amplitude on the order of $1 \mathrm{~V}$. With the increase of the gas pressure, the oscillations transform into the narrow-band spectrum type. The characteristic frequencies of those oscillations are almost linear functions of the pressure, as shown in Fig. 3. The frequencies weakly depend on the discharge current. The amplitude of oscillation can reach $30 \mathrm{~V}$ with a $100 \%$ modulation of the discharge current and voltage as it is shown in Fig. 4. Further increase of the gas pressure, higher than 10 Torr, leads to transformation of the plasma with nonlocal EEDF to plasma with local EEDF $\left(L>\lambda_{\varepsilon} \approx 10 / p\right.$ $\mathrm{cm}$ ) and that could lead to the complete disappearance of the oscillations.

The nature of the oscillation modifications with discharge parameters can be clarified by the differences in the processes of the plasma production and loss. The main processes of the plasma production are direct and stepwise ionizations of the helium atoms. Under the studied conditions, the charged particle loss is mainly due to their diffusion to the walls or electrodes, and then recombination on the walls. Slow, thermal electrons cannot reach the walls and cathode (due to the high negative potentials) and can move to the anode only. Their current to the anode is restricted by the near-anode potential

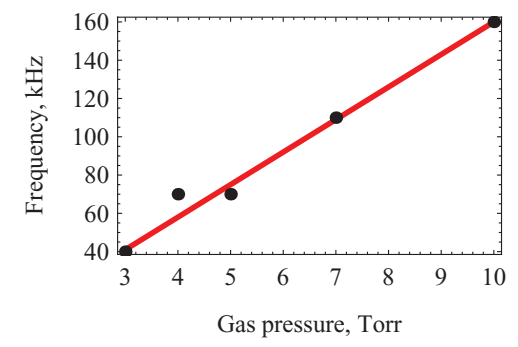

FIG. 3. Characteristic frequencies of the discharge voltage oscillations with respect to the gas pressures in helium discharge. 


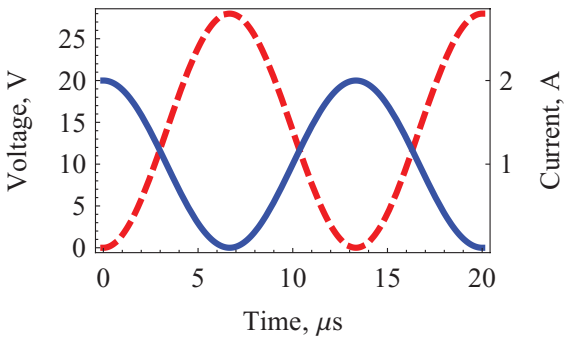

FIG. 4. Typical voltage (dashed curve) and current (solid curve) oscillations. Gas pressure is 5 Torr.

drop. In the limit of zero near-anode potential drop, the electron current of slow electrons is given by

$$
I_{c h}=\frac{1}{4} e N_{e} V_{T} S,
$$

where $e$ is the electron charge, $N_{e}$ is the electron density near the anode, $V_{T}$ is the thermal speed of electrons, and $S$ is the area of anode. For example, for the helium gas pressure of 5 Torr, $N_{e}=10^{11} \mathrm{~cm}^{-3}$ and $T_{e}=1 \mathrm{eV}$, Eq. (1) gives $I_{c h}$ $\approx 1.7 \mathrm{~A}$, whereas the experimental value is $I_{m}=2 \mathrm{~A}$ (see Fig. 4). The estimated ion drift time in the discharge gap is an order of several milliseconds, which is in a good agreement with frequencies of the oscillations. Disappearance of the oscillations for the gas pressures of higher than 10 Torr suggest that the oscillations are the feature of the nonlocal regime only $\left(L>\lambda_{\varepsilon} \approx 10 / p \mathrm{~cm}\right)$.

The collection of the anode current to the auxiliary electrode leads to a transformation of the discharge differential resistance from the negative to the positive one, as evident in Fig. 2 from curves 4, 5, and 6 . Curves 4 and 5 were obtained for the helium gas pressure of 1 Torr and curve 6 is for 4 Torr. Each curve was obtained with fixed auxiliary electrode current. The cathode current is the sum of the anode current and the auxiliary electrode current. The experimental data show that an increase in the current drawn to the auxiliary electrode makes discharge differential resistance even more positive (compare curves 4 and 5 in Fig. 2). In all cases with the positive differential resistance, the oscillations are suppressed and are practically absent.

Thus, small openings in boundaries of nonlocal plasma allow the subtraction of slow electrons while practically not affecting energetic plasma electrons. This allows transformation of the negative discharge differential resistance to positive and effective suppression of plasma oscillations and instabilities.

The authors are grateful to Maria Demidova for technical assistance. This research was supported by the DOE OFES (Contract No. DE-SC0001939) and the AFOSR. The work of V.I.D. was supported through the AF Summer Fellowship program.

\footnotetext{
${ }^{1}$ M. A. Lieberman and A. J. Lichtenberg, Principles of Plasma Discharge and Material Processing (Wiley, New York, 2005).

${ }^{2}$ L. D. Tsendin, Phys.-Uspekhi 53, 139 (2010).

${ }^{3}$ I. D. Kaganovich, V. I. Demidov, S. F. Adams, and Y. Raitses, Plasma Phys. Controlled Fusion 51, 124003 (2009).

${ }^{4}$ C. A. DeJoseph, Jr., V. I. Demidov, and A. A. Kudryavtsev, Phys. Plasmas 14, 057101 (2007); IEEE Trans. Plasma Sci. 34, 825 (2006).

${ }^{5}$ V. I. Demidov, C. A. DeJoseph, Jr., and A. A. Kudryavtsev, Phys. Rev. Lett. 95, 215002 (2005).

${ }^{6}$ V. I. Kolobov and L. D. Tsendin, Phys. Rev. A 46, 7837 (1992).

${ }^{7}$ S. A. Al-Bataineh et al., Plasma Processes Polym. 9, 638 (2012).

${ }^{8}$ T. Higashiguchi et al., J. Appl. Phys. 109, 013301 (2011).

${ }^{9}$ C. K. Eun and Y. B. Gianchandani, IEEE J. Quantum Electron. 48(6), 814 (2012).

${ }^{10}$ V. I. Demidov, S. F. Adams, J. Blessington, M. E. Koepke, and J. M. Williamson, Contrib. Plasma Phys. 50, 808 (2010).

${ }^{11}$ K. F. Chen and J. G. Eden, Appl. Phys. Lett. 93, 161501 (2008).

${ }^{12}$ Y. P. Raizer, Gas Discharge Physics (Springer, New York, 1997).

${ }^{13}$ Z. Lj. Petrovic, I. Stefanovic, S. Vrhovac, and J. Zivkovic, J. Phys. IV 7, C4-341 (1997).

${ }^{14}$ A. V. Phelps et al., Phys. Rev. E 47, 2825 (1993).

${ }^{15}$ I. Stefanovich et al., J. Appl. Phys. 110, 083310 (2011).

${ }^{16}$ F. Greiner, T. Klinger, A. Rohde, and A. Piel, Phys. Plasmas 2, 1822 (1995).

${ }^{17}$ F. Greiner, T. Klinger, and A. Piel, Phys. Plasmas 2, 1810 (1995).

${ }^{18}$ M. A. Fedotov, I. Kaganovich, and L. D. Tsendin, Sov. Phys. Tech. Phys. 39, 241 (1994).

${ }^{19}$ V. A. Godyak and V. I. Demidov, J. Phys. D: Appl. Phys. 44, 233001 (2011).

${ }^{20}$ V. I. Demidov, S. V. Ratynskaia, and K. Rypdal, Rev. Sci. Instrum. 73, 3109 (2002).
} 